\title{
Tomographic-like reconstruction of the percolation cluster as a phase transition
}

\author{
Nira Shimoni, Doron Azulai, Isaac Balberg, and Oded Millof \\ Racah Institute of Physics, The Hebrew University, Jerusalem 91904, Israel
}

\begin{abstract}
We observed a phase transition-like behavior that is marked by the onset of the realization of the connectivity between two sites on a two-dimensional cross-section of a three-dimensional percolation cluster. This was found using contact-resistance atomic force microscopy on carbon black/polymer composites. The features in the current images, when presented as a function of the cut-off current, or as a function of the total area covered by the electrically connected objects, appear to obey a cluster statistics that is similar to the one predicted and observed in continuum percolation systems.
\end{abstract}

PACS numbers: 72.80.Tm, 81.05.Qk, 68.35.-p, 64.60.Ak

While the basic theory of percolation seems to be well understood, there are many aspects of classical percolation theory that have not been investigated intensively in spite of their fundamental importance and practical applications. In particular, there is a group of problems associated with the fact that usually one observes three-dimensional (3D) structures with methods that yield two-dimensional images. Such are the projection, 2 the illumination $\mathrm{B}$ and the cross-section 1 of $3 \mathrm{D}$ percolation systems. Conspicuous application are astrophysical maps and hydrologica 10 information, and the determination of the structure of the phase in binary inhomogeneous media 1 . 0.11 .12 .13 In particular, it was only very recently that the dynamic properties associated with these problems have been considered 10 This was the phenomenon of flow between two surface sites, that are connected by the underlying 3D percolation cluster. In this paper we report a manifestation of the above-mentioned problems in binary composites using an experimental method that depends on the dynamical aspects of flow within a percolation cluster. As we show below, the connection between the geometrical and dynamical aspects can be described by a percolation-type phase-transitionlike behavior.

While manifestations of $3 \mathrm{D}$ properties in $2 \mathrm{D}$ data was found a long time agd 13 (on composites in which the electrifal properties have indicated a percolation behavior 4, 15) the more recent development of atomic force microsfopy (AFM) and its extensions, to stiffness microscopy 1 electrical force microscopy (EFM) 11 and contact-resistance microscopy 12 (C-AFM), have enabled more direct cross-section images of the percolation cluster. In particular, EFM images have been used for the experimental determination of the fractal dimension, 16 $D_{f}$, of the 3D percolating conducting phase in composites made of carbon black (CB) particles embedded in an insulating polymer 11

The initial aim of our study was to reexamine the above EFM results using the superior 12 C-AFM method, since the results obtained in Ref. 11 were subjected to a significant uncertainty yielding 17 a $D_{f}$ value in the range 2.6 \pm 0.6 . This permits a $D_{f}=3$ value, which is in the homogeneous (3D) regime, and thus may shed doubt as to the validity of the underlying scaling assumption claimed in Ref. 11 (see below). The latter spould yield the value borne out from percolation theory, $111 D_{f}=2.53$.

Our samples, with CB fillings between 10 and 23 vol\%, were prepared from a commercial grade polyethylene and Vulcan XC-72 carbon black 15. This CB has 100-1000 nmlong aggregates that consist of fused spherical graphite particles that are $30 \mathrm{~mm}$ in diameter14.18. The composites were prepared 11 in a common compounding and molding procedure 1920 resulting in uniform plaques 0.25 $\mathrm{mm}$ thick. The plaques were cut to long strips for the macroscopic measurements, described previously, 15 and to squares of $12 \times 12 \mathrm{~mm}^{2}$ for the present C-AFM study. A $5 \mathrm{~mm}$ wide silver-paint strip at the sample side served as a counter electrode to the local contact provided by the conducting tip. The C-AFM images were acquired more than $2 \mathrm{~mm}$ away from the strip, thus the current between the tip and the electrode is possible only if there is an "infinite" conducting cluster in the system, i.e., the current must flow between the tip and a branch of this infinite cluster. We call a cross section of such a branch, as reflected in the surface image, a "conducting island".

Our measurements were performed using a commercial (NT-MDT Solver) Scanning Force Microscope in the constant-force mode, with $0.03 \mathrm{~N} / \mathrm{m}$ cantilever stiffness. The current maps were measured along with the topography for a given tip-electrode voltage, $V$. The silicon cantilever tips had curvatures of less than $35 \mathrm{~nm}$, and were coated by a $25 \mathrm{~nm}$-thick titanium-nitride film. We thus estimate the resolution in the topographic images to be better than $60 \mathrm{~nm}$. The spatial resolution of the current maps is, however, much better, and was limited by our pixel size, which is of the order of $10 \times 10 \mathrm{~nm}^{2}$. This is important considering the size of the conducting particles (the CB aggregates, see above), since this means that we can assume that a pixel in an image belongs to a single particle. The noise level was around $0.02 \mathrm{nA}$ in most measurements and thus in our image analysis we did not consider currents below $0.04 \mathrm{nA}$. The images discriminate between different current intensities (between and within islands) and thus enable statistical analysis of the current images taken with a constant voltage. In particular, we extract from each image the individual and inclusive areas of the islands for currents larger than a 

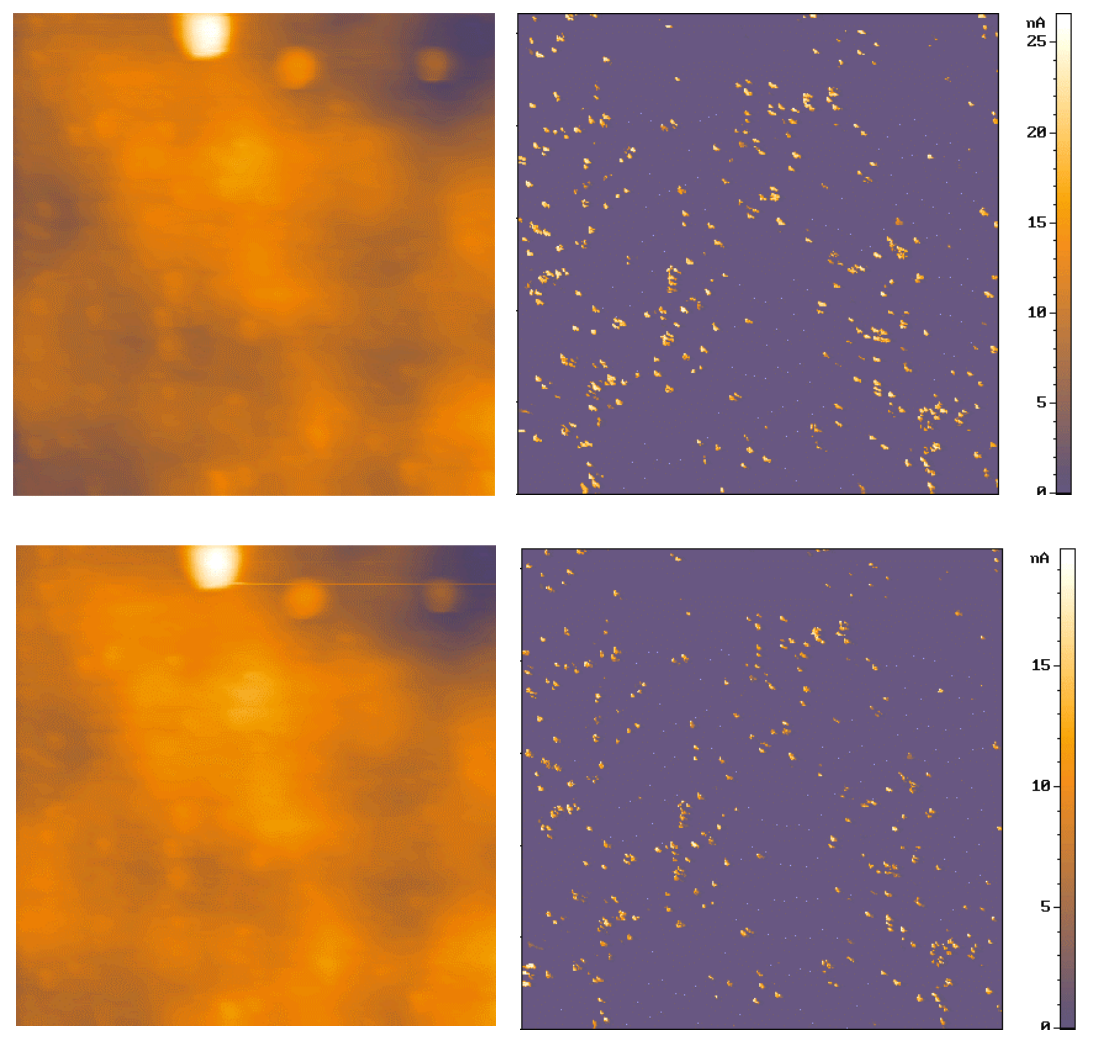

FIG. 1: $5 \times 5 \mu \mathrm{m}^{2}$ topographic (left) and current (right) images for a sample of 15 vol\% CB filling, obtained for two values of applied voltage, $V=1.8 \mathrm{~V}$ (lower scan) and $V=2.8 \mathrm{~V}$ (upper scan). The color scale range in the topographic images is 200 $\mathrm{nm}$, while in the current images it is $20 \mathrm{nA}$ and $27 \mathrm{nA}$ for the $1.8 \mathrm{~V}$ and $2.8 \mathrm{~V}$ scans, respectively.

chosen cutoff current $I_{\mathrm{CO}}$.

Turning to the results, in Fig. 1 we show typical topographic and current images obtained by the C-AFM for two values of $V$. We found no correlation between the surface morphology and the distribution of the "conducting islands". This implies that the current maps reflect mainly the bulk sample properties, namely, the 3D percolation cluster. The increase of the number of islands and their individual areas with $V$ underlines the 3D nature of the system as more percolation cluster branches can be detected with increasing $V$. The increase of the bias appears then to be associated with "deeper" cross sectioning of the conducting network of the bulk.

To check the fractal dimension of the islands area on the surface, $D_{s}$, we first considered samples with different $\mathrm{CB}$ contents. We measured the area covered by all the islands, $A_{t}$, as a function of the scanned area ("the window"), $L^{2}$. This was done first in the regime for which scaling behavior is expected 1 i.e., $\nu=10$ vol\%, which is close to the percolation threshold, $\nu_{c}(=9.3$ vol\% $)$ (15), and $L$ larger than the particle size $(\sim 0.5 \mu \mathrm{m}$ in our case) but smaller than the correlation length (which is of macroscopic order near the percolation threshold 12 say, larger than $10 \mu \mathrm{m})$. The results of our measurements for various cutoff currents are shown in Fig. 2.

For all nine cutoff currents considered (in the range $0.04 \leq I_{\mathrm{CO}} \leq 20 \mathrm{nA}$ ), the relation $A_{t} \propto L^{D_{s}}$ (expected from percolation theoryt) was obeyed, as seen in Fig. 2 for four cutoff currents. The extracted exponent for each of the nine cutoff currents has a value within the interval $1.35 \leq D_{s} \leq 1.65$. Our $D_{s}=1.5 \pm 0.15$ exponent has the same value as that obtained 110 from EFM measurements, but it has, as explained above, a higher certainty which is very significant. Utilizing the expectation 11.16 that the fractal dimension of the bulk cluster is given by $D_{f}=D_{s}+1$, we find that $D_{f}=2.50 \pm 0.15$, in agreement with the value expected for a $3 \mathrm{D}$ percolation cluster, 2.53. We have further established the validity of the above values, in addition to the smaller error bars and the fact that they were found for nine different cutoff currents, by an even more significant test. This is by performing the same measurements on a sample with $\nu$ well removed from $\nu_{c}$, where the "normal" 3D dimensionality should be recovered. Three cutoff currents $(0.5$, 4 , and $20 \mathrm{nA}$ ) were used in a measurement of a $\nu=15$ vol\% sample, all yielding a value of $D_{s}=2.00 \pm 0.05$. The clear distinction between the results for the $10 \mathrm{vol} \%$, and the 15 vol\% samples is very convincing evidence 


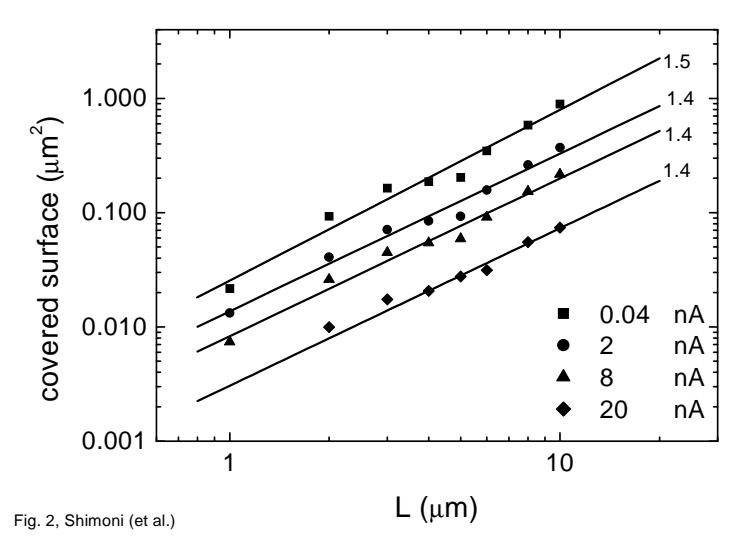

FIG. 2: The measured total area covered by "conducting islands", $A_{t}$, as a function of the square window size $L$ for a sample with 10 vol\% CB filling, for four different cutoff currents. The lines are fits to the scaling dependence, $A_{t} \sim L^{D_{s}}$, with the indicated $D_{s}$ values. The voltage applied in this case was $2 \mathrm{~V}$. The points in this and in other graphs represent mean values of data obtained for various samples and scans.

that our surface measurements reveal properties of the bulk percolation cluster. This behavior appears to be general, as it found not only for the present "highstructure" $14,15,18 \mathrm{CB} /$ polymer composite but also for another, "low-stfucture", CB/polymer composite that we have studied15, as well as for granular $\mathrm{Ni} / \mathrm{SiO}_{2}$ films. The $\mathrm{CB}$ system, however, is of particular interest, since it is not a "true" percolation system, in the sense that conduction takes place via tunneling between the CB particles even in the percolation cluster. Nevertheless, it does conform with $3 \mathrm{D}$ continuum percolation behavior 21

The results shown in Fig. 2 have significant implications beyond the latter conclusion. We see that, systematically, the lower the value of $I_{\mathrm{co}}$ the larger the value of $A_{t}$, for the same $V$ and $L$. Let us consider this significance. All the conducting islands on the surface belong to the percolation cluster, as otherwise, no current can flow between them and the counter-electrode. On the other hand, the value of $I_{\mathrm{CO}}$ determines the corresponding longest possible current paths that can participate in the conduction (see below). We can then interpret the values of $I_{\mathrm{CO}}$ in terms of connectivity. The higher the $I_{\mathrm{CO}}$, the smaller the ensemble of conductance paths between the observed islands and the electrode via the percolation cluster. But then, two islands that are seen at a given $I_{\mathrm{CO}}$ are also connected among themselves via the $3 \mathrm{D}$ percolation cluster. The lower the value of $I_{\mathrm{CO}}$, the longer the conductance paths included in the ensemble of possible paths and the larger the number of detected conducting islands that are interconnected via the percolation cluster. Thus the variation of $I_{\mathrm{C} o}$ acts as an electrical cross-sectioning parameter of the $3 D$ percolation cluster. In other words, the lower the value of $I_{\mathrm{co}}$, the "deeper" the (electrically) added "tomographic-like" section of this cluster. So, while it is obvious that all the

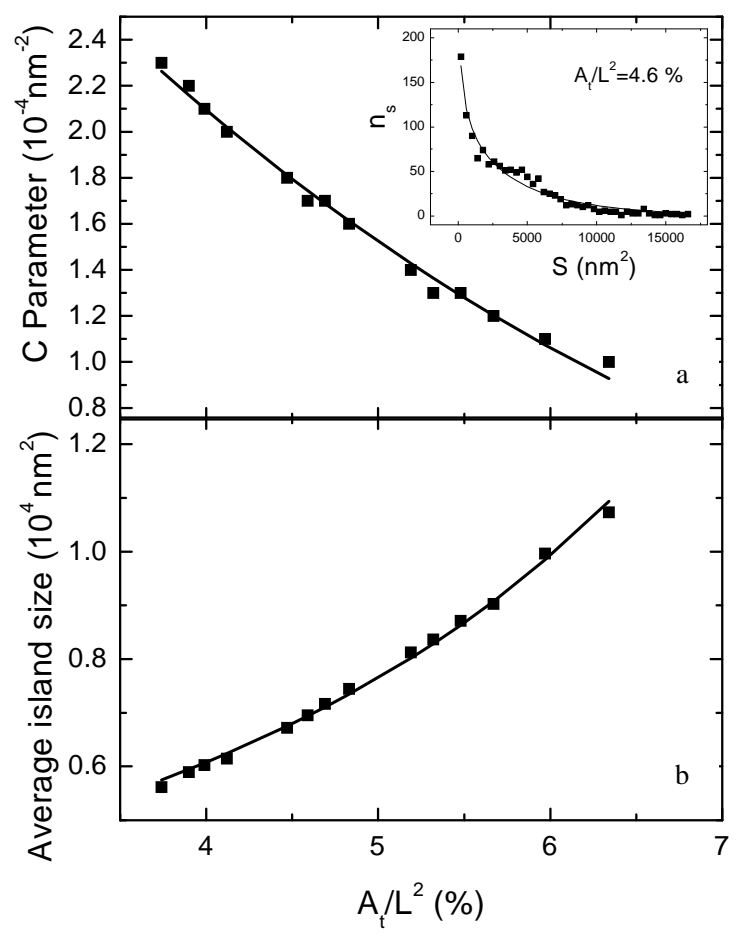

Fig. 3, Shimoni et al.

FIG. 3: (a) The dependence of the parameter $C$ on the fractional total covered area, $A_{t} / L^{2}$. Inset: the number of observed islands as a function of their size for $A_{t} / L^{2}=4.6 \%$. (b) The dependence of the average size of the observed "conducting island" on the fractional total covered area, $A_{t} / L^{2}$. The measurements were carried out for $\nu=15 \mathrm{vol} \%, L=10 \mu \mathrm{m}$ and $V=2 \mathrm{~V}$. The lines present fits to percolation theory-like expressions, see text.

conducting islands are connected, the electrical realization of this connectedness is limited in our measurements to part of the conductance paths in the system. This interpretation gives a meaning to the $I_{\mathrm{CO}} \rightarrow 0$ limit as the $I_{\mathrm{CO}}$ value for which all available connecting paths between two islands are realized. We have then a transition from a partially realized (via the $3 \mathrm{D}$ percolation cluster) connectivity of the islands in the system to a fully realized connectivity of this system. Our experimental approach here is closely related to the well-known concepts of optimal and shortest paths. 22 In fact, the higher the chosen current cutoff, the closer the collection of current paths to the shortest electrical (and statistically, probably also the geometrical "chemical distance" 22 ) path. Our $I_{\text {co values }}$ then represent a hierarchy of collection of paths that deviate from the shortest electrical path. The $I_{\mathrm{co}} \rightarrow 0$ limit is associated with the collection of all possible paths.

Can the above onset of realized connectivity at $I_{\mathrm{CO}} \rightarrow 0$ be described as a percolation-type phase transition? A priori there is a difficulty in trying to examine this question, since we do not know the distribution of conductance paths in order to apply $I_{\mathrm{CO}}$ as a "thermodynamic parameter" 23 of a phase transition. However, there is an obvious parameter that $I_{\text {co }}$ can be mapped to (see 
Fig. 2) and with which we are familiar from the theory of continuum percolation 21 22 24. This parameter is the fractional "covered" or "occupied" area $A_{t} / L^{2}$ that can be simply mapped onto the site occupation probability $p$ in lattice percolation 21. The choice of $A_{t} / L^{2}$ as a "thermodynamic parameter" that describes the cross sectioning mentioned above also seems to suggest the value of its critical point, $A_{t c} / L^{2}$, as the value of $A_{t} / L^{2}$ when $I_{\mathrm{CO}} \rightarrow 0$. We point out again that the value of $A_{t c} / L^{2}$ does not yield any particular feature in the observed image, and that the transition is "hidden" here by the underlying $3 \mathrm{D}$ percolation cluster. The parameter $\left(A_{t}-A_{t c}\right) / L^{2}$ may then be, as in lattice or continuum percolation, the parameter that characterizes the proximity to the critical point 21 24. Having this parameter, it was natural to determine if cluster statistics such as in phase transitions in 23 and in continuum percolation in particular, 1.22 24 will be observed in the present case. For that purpose we examined the behavior of two quantities, that can be readily monitored and analyzed. To find whether they show the behavior of a "phase transition" we have to chect if they obey the corresponding power-law behaviorst22 23 for the same $A_{t c}$.

Following the above considerations we have plotted the number of observed islands, $n_{s}$, as a function of the island area, $S$, and we found that, as shown in the inset of Fig. 3(a) for $A_{t} / L^{2}=4.6 \%$, the results can be well fitted by a ${ }^{\propto} S^{-\tau} \exp (-C S)$ behavior (as in percolation theory 1,22$)$. The specific prediction of percolation theory is that $C \propto\left(A_{t}-A_{t c}\right)^{1 / \sigma}$ where $\tau$ and $1 / \sigma$ are dimensional-dependent exponents. As shown in Fig. $3(\mathrm{a})$, very good fits to the data with that dependence are achieved for a sample of $\nu=15 \mathrm{vol} \%$. The best fit yielded $A_{t c} / L^{2}=13 \%, \tau=0.25$ and $1 / \sigma=2.65$. An analysis of different areas of this same sample yielded $A_{t c} / L^{2}$ values in the range of $13 \pm 1$ results were obtained for other values of $\nu$, and the corresponding values of $A_{t c}$ were found to increase with $\nu$.

The other dependence that we evaluated was that of the average size of the conducting islands (the cluster size in percolation theory 22$)<S>$ on the above proximity parameter. $\langle S\rangle$ is defined in percolation theory as $\Sigma n_{s} S^{2} / \Sigma n_{s} S$, and is predicted to behave as $\left(A_{t}-A_{t c}\right)^{-\gamma}$. We checked then whether the data obtained for $\langle S\rangle$ can be fitted by that power-law. The best fit for the 15 vol\% sample yielded $\gamma=1.9$ and, again, $A_{t c} / L^{2}=13 \%$. This is shown in Fig. 3(b).

The important conclusion that can be derived from Fig. 3 is that the dependencies of $C$ and $\langle S\rangle$ on $\left(A_{t}-A_{t c}\right)$ can be fitted very well to a power law, both with the same $A_{t c}$ and with percolation theory-like exponents. The quality of the data is not sufficient for obtaining exact values of the exponents, but the fact that the same $A_{t c}$ value is found with reasonable exponents does establish that we have here a behavior that can be well described as a phase transition. The interesting finding here is that while we do not have any "visible" phase transition in the image (such as, say, 2D connectivity), the quantity $A_{t}$ (or the hidden variable $I_{\mathrm{co}}$ ) serves as the "thermodynamic parameter" of the phase transition in the realization of the connectivity of the underlying 3D system. The dynamic aspects, emphasized here by the dependence of the connectivity on $V$, are similar to the conduction under constant pressure differepge in the case of flow between two sites in porous media.10 In fact, as will be described elsewhere, the values of $A_{t}$ that are determined for different values of $V$ exhibit a similar behavior to the one described here for different values of $I_{\mathrm{CO}}$.

\section{Acknowledgments}

The authors would like to thank M. B. Heaney and M. Wartenberg for the samples used in this study and A. Aharony, K. Schwartz and A. Drory for helpful discussions. This work was supported by the Israel Science Foundation and the Niedersachsen Foundation.
* Electronic address: milode@vms.huji.ac.il

1 D. Stauffer and A. Aharony, Introduction to Percolation Theory (Taylor and Francis, London, 1992).

2 See for example, A.E. Pashitski, et al., Physica C 246, 133 (1995).

3 See for example, A. Margolina and M. Rosso, J. Phys. A. Math. Gen. 25, 3901 (1992).

4 See, for example, J. Planes, Y. Samson and Y. Chaguettine, Appl. Phys. Lett. 75, 1395 (1999).

5 See for example, A. Davis and P. Coles, Mons. Not. R. Astron. Soc. 260, 553 (1993); M. Beech, Astrophys. Space. Sci. 192, 103 (1992).

${ }^{6}$ K.L. Nielsen, J.P. Lynch and H.N. Weiss, Am. J. Bot. 84, 26 (1997).

7 L. Porthu et al., J. Bone. Miner. Res. 14:Sup 486 (1999), and K. Uemura et al., J. Nucl. Med. 38, 883 (1997).
8 A. Louche, et al., Solar Energy 46, 255 (1991).

${ }^{9}$ V. Nikora, R. Ibbitt and U. Shankar, Water Resour. Res. 323375 (1996).

10 J.S. Andrade, Jr., et al. Phys. Rev. E, 62, 8270 (2000).

11 R. Viswanathan and M.B. Heaney, Phys. Rev. Lett. 75, 4433 (1995).

12 J. Ravier et al., Carbon 39, 287 (2001), and J. Ravier, Thesis, University of Bordeaux, I, 2001.

13 I. Balberg and J. Blanc, Phys. Rev. B. 31, 8295 (1985).

14 I. Balberg, Phys. Rev. Lett. 59, 1305 (1987).

15 Z. Rubin et al., Phys. Rev. B 59, 12196 (1999), and references therein.

16 B.B. Mandelbrot, The Fractal Geometery of Nature (Freeman, New York, 1977).

17 R. Viswanathan and M.B. Heaney, Phys. Rev. Lett. 76, 3661 (1996). 
18 For a review on Carbon Blacks and their composites see, Carbon Black, J.B. Donnet, R.L. Bansal and M.-J. Wang, editors (Dekker, New York, 1993).

19 S. H. Foulger, J. of Appl. Poly. Science 72, 1573 (1999).

20 M.B. Heaney, Appl. Phys. Lett. 69, 2602 (1996).

${ }^{21}$ For reviews, see for example, I. Balberg, Phil. Mag. B 56, 991 (1987), and I. Balberg, Carbon 40, 139 (2002).

22 For a review see, A. Bunde and S. Havlin (eds.), Fractals and Disordered Systems, 2nd. Ed. (Springer, New York,
1996); for more recent works see for example N. Schwartz, A.L. Nazaryev and S. Havlin, Phys. Rev. E. 58, 7642 (1998) and N.V. Dokholyan et al., J. Stat. Phys. 93, 603 (1998).

23 H.E. Stanley, Introduction to Phase Transitions and Critical Phenomena (Clarendon Press, Oxford, 1971).

24 I. Balberg and N. Binenbaum, Phys. Rev. A 31, 1222 (1985). 